\title{
Two reconstructors for M-channel time- interleaved ADCs with missing samples
}

Anu Kalidas Muralidharan Pillai and Håkan Johansson

Linköping University Post Print

Tweet

N.B.: When citing this work, cite the original article.

(C2014 IEEE. Personal use of this material is permitted. However, permission to reprint/republish this material for advertising or promotional purposes or for creating new collective works for resale or redistribution to servers or lists, or to reuse any copyrighted component of this work in other works must be obtained from the IEEE.

Anu Kalidas Muralidharan Pillai and Håkan Johansson, Two reconstructors for M-channel time-interleaved ADCs with missing samples, 2014, 12th IEEE International New Circuits and Systems Conference (NEWCAS), Trois-Rivières, Canada.

Postprint available at: Linköping University Electronic Press http://urn.kb.se/resolve?urn=urn:nbn:se:liu:diva-106048 


\title{
Two Reconstructors for $M$-Channel Time-Interleaved ADCs with Missing Samples
}

\author{
Anu Kalidas Muralidharan Pillai and Håkan Johansson \\ Division of Electronics Systems, Department of Electrical Engineering, Linköping University, SE-581 83, Sweden \\ Email: \{kalidas, hakanj\}@isy.liu.se
}

\begin{abstract}
In this paper, we explore two nonrecursive reconstructors which recover the uniform-grid samples from the output of a timeinterleaved analog-to-digital converter (TI-ADC) that uses some of the sampling instants for estimating the mismatches in the TI-ADC. Nonuniform sampling occurs due to timing mismatches between the individual channel ADCs and also due to missing input samples. Compared to a previous solution, the reconstructors presented here offer substantially lower computational complexity.
\end{abstract}

\section{INTRODUCTION}

Time-interleaved analog-to-digital converters (TI-ADCs) make use of multiple ADCs in parallel. The output of the TI-ADC is formed by interleaving the outputs from all the channel ADCs. In an $M$ channel TI-ADC, the channel ADCs operate at a rate which is $M$ times lower than the output rate of the TI-ADC. This helps to reduce the requirements on the individual channel ADCs. Sampling clocks to the channel ADCs are uniformly skewed with respect to each other so that at any sampling instant, the input is sampled by only one channel ADC. In practice, due to non-idealities, the time skews between the sampling clocks will not be uniform. Due to these static timeskew errors, the TI-ADC output will be a periodically nonuniformly sampled version of the input. This degrades the performance at the output of the TI-ADC.

One way to alleviate the effect of time-skew errors is through digital calibration at the output of the TI-ADC. Digital calibration involves both estimating the time-skew errors as well as reconstructing the uniform-grid samples based on the estimate. Estimators can be broadly classified into foreground or background techniques. Even though estimators employing foreground techniques achieve better convergence compared to those using background techniques, the former estimators interrupt the normal operation of the TI-ADC. Recently, an iterative background estimation scheme that achieves faster convergence compared to other background estimation schemes was proposed in [1]. In this scheme, a known calibration signal is injected at predefined sampling instants. The time-skew errors are then estimated by using the TI-ADC output samples corresponding to the calibration signal. The sampling instants for injecting the calibration signal are selected such that the sampled calibration signal contains output samples from all the channel ADCs. Since the TIADC input is not sampled at the sampling instants reserved for the calibration signal, the sampled signal at the TI-ADC output will contain missing samples. Thus, the estimation scheme in [1] requires a reconstructor that should recover the missing samples in addition to compensating for the time-skew errors. An iterative reconstruction scheme with better convergence rate was proposed in [1]. However, the computational complexity, measured in terms of the number of multiplications per corrected output sample, is very high for this scheme. Also, the reconstructor in [1] uses recursive structures which can lead to stability issues as well as limit the maximal data rate [2]. In this paper, we focus on the reconstruction part and introduce nonrecursive reconstructors with a substantially lower computational complexity than that of [1]. Since digital compensation of gain and offset mismatches between the channel ADCs is simple, here, we only consider reconstructors that compensate for timing mismatches between the channel ADCs. Also, we assume that the operating frequencies and resolution are not very high due to which it can be considered that the channel ADCs suffer only from static time-skew errors [3].

Several papers, including [4]-[6], have addressed the problem of using nonrecursive structures for the reconstruction of periodically nonuniformly sampled signals without any missing samples. In this paper, we explore two non-iterative and nonrecursive finite-length impulse response (FIR) reconstructors which, in addition to correcting the time-skew errors, can also recover the missing samples. For a given set of time-skew errors, the first reconstruction scheme achieves minimal order and implementation complexity. This scheme utilizes the time-varying FIR reconstructor introduced in [6], but here some of the impulse response taps are zero due to the missing samples. We also present a least-squares design for the proposed reconstructor. Even though the design procedure is similar to that of the timevarying reconstructor in [6], the difference arises because of the zero-valued taps. The second scheme presented here is a sub-band based reconfigurable reconstructor proposed recently in [7]. While the implementation complexity of the sub-band based reconstructor is higher than that of the first scheme, it has lower online redesign complexity. In order to be able to provide a fair comparison between the two schemes, we also provide a least-squares design of the subband based reconstructor which was not presented in [7].

\section{BACKGROUND}

In an $M$-channel TI-ADC, a uniform time skew between the sampling clocks of the individual channel ADCs is required to ensure that the TI-ADC output $x(n), n \in \mathbb{Z}$, is a uniformly sampled version of the input $x_{a}(t)$. If $T$ represents the sampling period of the TI-ADC, $x(n)=x_{a}(n T)$. In practice, due to timing mismatches between the individual channel ADCs, the input $x_{a}(t)$ is nonuniformly sampled by the TI-ADC. Assume that $\varepsilon_{n} T$ represents the difference between the ideal sampling instant $n T$ and the corresponding actual sampling instant. Then, the nonuniformly sampled signal at the output of the the TI-ADC is $v(n)=x_{a}\left(n T+\varepsilon_{n} T\right)$. Hereafter, for simplicity, we will assume that $T=1$. Since the output of an $M$-channel TI-ADC is formed by interleaving the outputs of $M$ channel ADCs, $\varepsilon_{n}=\varepsilon_{n+M}$. This implies that $v(n)$ is a periodically nonuniformly sampled version of the input $x_{a}(t)$.

Reconstructors are used to recover the uniform-grid samples $x(n)$ from $v(n)$. However, in order to recover $x(n)$ from $v(n)$, the time-skew errors $\varepsilon_{n}$ should be estimated. A background calibration technique in which some of the sampling instants are reserved for estimating the mismatches in the TI-ADC was proposed in [1]. A known calibration signal $c_{a}(t)$ is applied to the input of the TI-ADC at predefined sampling instants $t=r M_{c}-1, \forall r \in \mathbb{Z}$. The resulting nonuniformly 


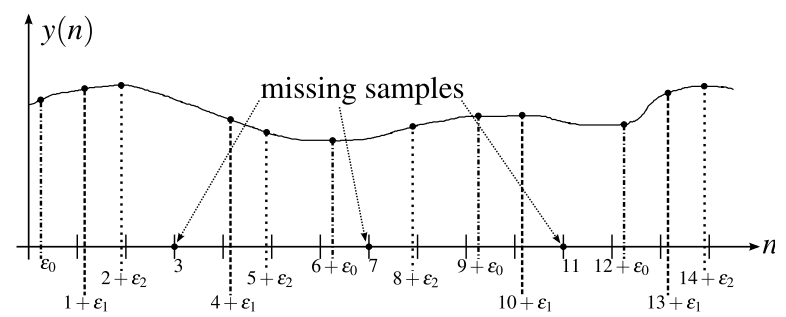

Fig. 1. Periodically nonuniformly sampled sequence at the output of a threechannel TI-ADC $(M=3)$ with missing samples due to sampling instants $\left(M_{C}=\right.$ 4) reserved for a calibration signal.

sampled calibration signal $c(r)$ is then compared with a known reference sequence, $c_{r e f}(r)=c_{a}\left(r M_{c}-1\right)$, to estimate $\varepsilon_{n}$. As the estimation requires samples from all the channel ADCs, $M_{c}$ is chosen such that $M_{c}$ and $M$ are co-prime. Compared to blind estimators, the estimation scheme in [1] achieves faster convergence rate. Since some of the sampling instants are reserved for the calibration signal, the input $x_{a}(t)$ is not sampled at sampling instants $t=r M_{c}-1$. Due to this, the input to the reconstructor $y(n)$ is given by

$$
y(n)= \begin{cases}0, & n=r M_{c}-1 \\ v(n), & \text { otherwise. }\end{cases}
$$

The reconstruction problem is thus to recover $x(n)$ from $y(n)$ given the values of $\varepsilon_{n}$.

\section{CONSTRAINED TIME-VARYING FIR RECONSTRUCTOR}

Due to missing samples at time instants $t=r M_{c}-1, y(n)$ in (1) is an $M M_{c}$-periodically nonuniformly sampled version of the input $x_{a}(t)$. This is exemplified Fig. 1 which shows the output $y(n)$ of a three-channel TI-ADC $(M=3)$ in which every fourth sample is used for the calibration signal $\left(M_{c}=4\right)$. Reconstruction of periodically nonuniformly sampled signals using general timevarying FIR reconstructors was studied in [6]. In such reconstructors, the reconstructed output $\widetilde{x}(n)$ is given by

$$
\widetilde{x}(n)=\sum_{k=-N_{h_{n}} / 2}^{N_{h_{n}} / 2} y(n-k) h_{n}(k)
$$

where $N_{h_{n}}$ is the order and $h_{n}(k)$ is centered at the sample that is to be recovered. For simplicity in the design, but without any loss of generality, it is assumed that $h_{n}(k)$ is noncausal. It is noted that the time-varying reconstructor $h_{n}(k)$ used to reconstruct an $N$-periodically nonuniformly sampled signals is also periodic with $h_{n}(k)=h_{n+N}(k)$. In the case of an $M$-channel TI-ADC where every $M_{c}$ th sampling instant is reserved for the calibration signal, $N=M M_{c}$.

Unlike the general time-varying FIR reconstructor in [6], here, the missing samples in $y(n)$ restrict some of the impulse response coefficients in $h_{n}(k)$ to be zero. Specifically,

$$
h_{n}(k)=0, \forall k=n-r M_{c}+1, r \in \mathbb{Z} .
$$

We call this reconstructor a constrained time-varying FIR reconstructor. Assume that for a given $n, R_{n}$ impulse response coefficients in $h_{n}(k)$ are non-zero. Let $\mathbf{h}_{n}=\left[h_{n}\left(k_{1}\right) h_{n}\left(k_{2}\right) \ldots h_{n}\left(k_{R_{n}}\right)\right]$ represent the vector containing the non-zero impulse response coefficients where $k_{i}, i=1,2, \ldots, R_{n}$ are the indices of the non-zero impulse response coefficients. Then, the reconstructed output $\widetilde{x}(n)$ is given by

$$
\widetilde{x}(n)=\sum_{i=1}^{R_{n}} y\left(n-k_{i}\right) h_{n}\left(k_{i}\right) .
$$

In order to implement practical filters, it is assumed that the signals are oversampled such that they are bandlimited to $|\omega| \leq \omega_{0}<\pi$. Like in [6], how well $\widetilde{x}(n)$, for all $n$, approximates the ideal uniformgrid samples $x(n)$ depends on the difference between $A_{n}(j \omega)$ and 1 , $n=0,1, \ldots, N-1$, where

$$
A_{n}(j \omega)=\sum_{i=1}^{R_{n}} h_{n}\left(k_{i}\right) e^{-j \omega\left(k_{i}-\varepsilon_{n-k_{i}}\right)} .
$$

\section{A. Least-Squares Design}

Here, we use a least-squares approach to determine the impulse response coefficients $h_{n}\left(k_{i}\right)$. For this purpose, we express the error power function

$$
\mathrm{P}_{n}=\frac{1}{2 \pi} \int_{-\omega_{0}}^{\omega_{0}}\left|A_{n}(j \omega)-1\right|^{2} \mathrm{~d} \omega
$$

in terms of $\mathbf{h}_{n}$. The nonzero impulse response coefficients $\mathbf{h}_{n}$ that minimizes $\mathrm{P}_{n}$ is then obtained by setting the partial derivative of $\mathrm{P}_{n}$ with respect to $\mathbf{h}_{n}$ to zero and then solving for $\mathbf{h}_{n}$. Then, $\mathbf{h}_{n}$ can be determined using a closed-form solution via matrix inversion.

Using a constrained time-varying FIR scheme for reconstructing the uniform-grid samples from an $M M_{c}$-periodically nonuniformly sampled signal requires $M M_{c}$ separate FIR filters. The order of each $h_{n}(k), n \in\left[0,1, \ldots, M M_{c}-1\right]$, depends on how close the value of a sample to be reconstructed is to the desired uniform-grid sample value. Thus, the FIR filter used to recover the missing sample requires the highest order. The implementation complexity of the reconstructor, measured in terms of the number of multiplications required per corrected output sample on average, is equal to $\sum_{n=0}^{M M_{c}-1} R_{n} / M M_{c}$. For a given set of time-skew errors and $M_{c}$, the overall order and implementation complexity is minimal for this reconstructor. However, if the time-skew errors change, the coefficients of all the $M M_{c}$ FIR filters have to be redetermined which requires $M M_{c}$ separate matrix inversions.

\section{SUB-BAND BASED RECONSTRUCTOR}

In [7], it was observed that the problem of reconstructing the nonuniformly sampled signal at the output of a TI-ADC with missing samples is similar to that of recovering the missing samples from a sub-Nyquist sampled sparse multi-band signal. Thus, it was shown that the reconstruction of an $N$-periodically nonuniformly sampled signal from an $M$-channel TI-ADC with missing samples can be performed using a set of $K=N-M$ channel analysis and synthesis filter banks. For this purpose, the whole Nyquist band was divided into $N$ sub-bands of equal length $\pi / N$ and where only the first $K$ bands are assumed to be active. In principle, each analysis filter $B_{k}\left(e^{j \omega}\right), k \in[1,2, \ldots, K]$, extracts the signals in one of the $K$ active sub-bands and also makes the extracted signals uniformly sampled by compensating for the channel time-skew errors. However, they are designed simultaneously ensure that the overall reconstructors covers the whole passband region $\omega \in\left[-\omega_{0}, \omega_{0}\right]$. Due to the missing samples, only the inputs to $K$ polyphase components of each analysis filter $B_{k}\left(e^{j \omega}\right)$ are non-zero. In a full-length paper under way [8], we show that the polyphase components $B_{k m_{\ell}}\left(e^{j \omega}\right), m_{\ell} \in[0,1, \ldots, N]$, $\ell=1,2, \ldots, K$, approximate generalized fractional-delay filters so that

$$
B_{k m_{\ell}}\left(e^{j \omega}\right) \approx \beta_{k m_{\ell}} e^{j\left(\omega\left(m_{\ell}+\varepsilon_{\ell}\right) / N+\alpha_{k m_{\ell}} \operatorname{sgn}(\omega)\right)}, \omega \in[-\pi, \pi] .
$$

In (7), $\beta_{k m_{\ell}}$ and $\alpha_{k m_{\ell}}$ are the modulus and angle, respectively, of a corresponding complex constant $c_{k m_{\ell}}$ which is determined using a matrix inversion [7].

The reconstruction scheme is shown in Fig. 2. In order to reduce the complexity of the online redesign block, the polyphase branches 


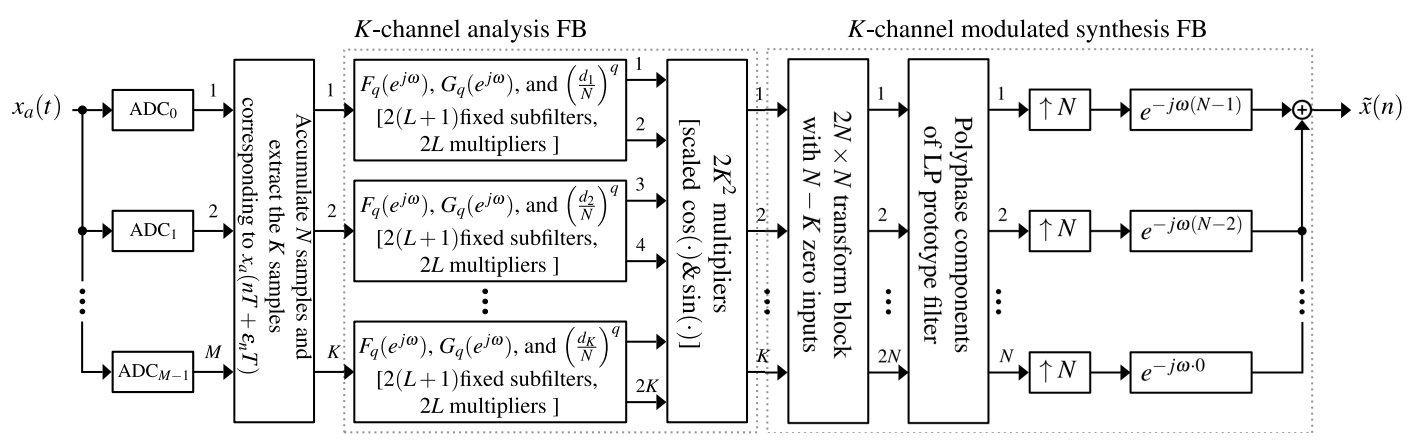

Fig. 2. Sub-band based reconstruction scheme.

of $B_{k}\left(e^{j \omega}\right)$ are implemented using the structure in [9]. Thus, the $B_{k m_{\ell}}\left(e^{j \omega}\right)$ are expressed using a common set of $L+1$ fixed subfilters $F_{q}\left(e^{j \omega}\right)$ and $G_{q}\left(e^{j \omega}\right), q=0,1, \ldots, L$, such that

$$
B_{k m_{\ell}}\left(e^{j \omega}\right)=\gamma_{k m_{\ell}} \sum_{q=0}^{L}\left(\frac{d_{\ell}}{N}\right)^{q} F_{q}\left(e^{j \omega}\right)+\zeta_{k m_{\ell}} \sum_{q=0}^{L}\left(\frac{d_{\ell}}{N}\right)^{q} G_{q}\left(e^{j \omega}\right)
$$

where $\gamma_{k m_{\ell}}=\beta_{k m_{\ell}} \cos \left(\theta_{k m_{\ell}}\right), \zeta_{k m_{\ell}}=\beta_{k m_{\ell}} \sin \left(\theta_{k m_{\ell}}\right), \theta_{k m_{\ell}}=\alpha_{k m_{\ell}}+$ $\pi / 4, d_{\ell}=m_{\ell}+\varepsilon_{\ell}$, and $N=M M_{c}$. The different polyphase branches can be obtained via different sets of values for $\gamma_{k m_{\ell}}, \zeta_{k m_{\ell}}$, and $d_{\ell}$. When the time-skew errors change, only the general multipliers corresponding to $\gamma_{k m_{\ell}}, \zeta_{k m_{\ell}}$, and $d_{\ell}$ need to be updated with the corresponding new values. Also, the new values of the constants $\gamma_{k m_{\ell}}$ and $\zeta_{k m_{\ell}}$ can be determined through a single $K \times K$ matrix inversion.

The bandpass filters $C_{k}\left(e^{j \omega}\right)$ in the synthesis filter bank (FB) are fixed and are implemented using a cosine-modulated FB. The prototype filter for the cosine-modulated FB is a lowpass filter with cutoff frequency at $\pi / 2 N$.

\section{A. Least-Squares Design}

First, the coefficients of the lowpass prototype filter for the cosinemodulated FB are determined and fixed. Next, the subfilters $F_{q}\left(e^{j \omega}\right)$ and $G_{q}\left(e^{j \omega}\right), q=0,1, \ldots, L$, are designed and fixed. Here, we present a least-squares approach for determining the coefficients of the fixed subfilters. For this purpose, we derive an appropriate error power function $P$ that can be minimized in the least-squares sense. Using the FB representation in Fig. 2, we can express the Fourier transform (FT) of the reconstructed output $\widetilde{x}(n)$ in terms of the FT of $x(n)$ according to

$$
\widetilde{X}\left(e^{j \omega}\right)=V_{0}\left(e^{j \omega}\right) X\left(e^{j \omega}\right)+\sum_{p=1}^{N-1} V_{p}\left(e^{j \omega}\right) X\left(e^{j(\omega-2 \pi p / N)}\right)
$$

where $V_{0}\left(e^{j \omega}\right)$ is the distortion function and $V_{p}\left(e^{j \omega}\right), \quad p=$ $1,2, \ldots, N-1$ are the aliasing functions with

$$
V_{p}\left(e^{j \omega}\right)=\frac{1}{N} \sum_{k=1}^{K} B_{k}\left(e^{j(\omega-2 \pi p / N)}\right) C_{k}\left(e^{j \omega}\right)
$$

for $p=0,1, \ldots, N-1$. From (9), it can be seen that, how closely the reconstructed signal $\widetilde{x}(n)$ resembles the uniformly sampled signal $x(n)$ depends on how well the distortion term and the aliasing terms in (9) approximate unity and zero, respectively, in the passband region $\omega \in\left[-\omega_{0}, \omega_{0}\right]$. Thus, we can express the error power function as

$$
\mathrm{P}=\sum_{p=0}^{N-1} \mathrm{P}_{p}
$$

where

$$
\mathrm{P}_{p}=\frac{1}{2 \pi} \int_{-\omega_{0}}^{\omega_{0}}\left|V_{p}\left(e^{j \omega}\right)-\operatorname{Des}_{p}\right|^{2} \mathrm{~d} \omega
$$

with $\operatorname{Des}_{0}=1$ and $\operatorname{Des}_{p}=0$ for $p=1,2, \ldots, N-1$.

Let the vectors $\mathbf{f}_{q}$ and $\mathbf{g}_{q}, q \in[0,1, \ldots, L]$, represent the impulse response coefficients of $F_{q}\left(e^{j \omega}\right)$ and $G_{q}\left(e^{j \omega}\right)$, respectively, and let the vector $\mathbf{h}=\left[\begin{array}{lllllll}\mathbf{f}_{0} & \mathbf{g}_{0} & \mathbf{f}_{1} & \mathbf{g}_{1} & \ldots & \mathbf{f}_{L} & \mathbf{g}_{L}\end{array}\right]$. Then, the impulse response coefficients of the subfilters $\mathbf{f}_{q}$ and $\mathbf{g}_{q}, q=0,1, \ldots, L$ can be determined by expressing $\mathrm{P}$ in terms of $\mathbf{h}$ and solving $\partial \mathrm{P} / \partial \mathbf{h}=0$ to get the $\mathbf{h}$ that minimizes P. Ideally, in order to ensure that the coefficients of the fixed subfilters can be used for all the possible combinations of $\varepsilon_{n}$, each $\mathrm{P}_{p}, p \in[0,1, \ldots, N-1]$, should be formed by integrating (12) over all the possible combinations $\varepsilon_{n}$. However, in practice, it is sufficient to compute each $\mathrm{P}_{p}$ by evaluating (12) for the worst combinations of $\varepsilon_{n}$ and adding them together. Like in the constrained time-varying FIR reconstructor, here also we can derive a closed form solution for $\partial \mathrm{P} / \partial \mathbf{h}=0$ which can then be computed using a matrix inversion.

\section{DESIGN EXAMPLES}

In this section, we compare the design and implementation complexities of the two nonrecursive reconstructors presented in Sections III and IV. For this purpose, we use the four-channel TI-ADC $(M=4)$ case considered in Example A in Section VI of [1]. Hence, it is assumed that the timing mismatches in the channel ADCs are $\varepsilon_{0}=0.01, \varepsilon_{1}=-0.05, \varepsilon_{2}=0.04$, and $\varepsilon_{3}=-0.03$, the bandwidth of the reconstructor, $\omega_{0}$, is $0.8 \pi$, and that every seventh sample is used by the estimator, i.e., $M_{c}=7$. Thus, for the proposed reconstructors, $N=28, K=24$, and the input sampling instants in the first period are $n=\{[0: 5],[7: 12],[14: 19],[21: 26]\}$. The reconstructors were designed such that, after reconstruction, all the distortion and aliasing terms are below $-50 \mathrm{~dB}$. This requires the error power measures defined in Sections III and IV to be below $-64 \mathrm{~dB}$. Also, this corresponds to three iterations of the reconstructor in [1] which is required to keep the spurs below $-50 \mathrm{~dB}$ as can be seen from Fig. 9(c) in [1] and from Fig. 3 which shows the spectrum before and after reconstruction using the constrained time-varying FIR reconstructor. Here, the constrained time-varying FIR reconstructor requires 28 different FIR filters. As can be seen from Table I, the order of the FIR filter is highest for the sampling instants that correspond to the missing samples $(n=6,13,20,27)$. The design and implementation complexities of a reconstructor using the constrained time-varying FIR scheme as well as the sub-band based scheme and the reconstruction scheme in [1] are tabulated in Table II. The values for the constrained time-varying FIR reconstructor indicate the lower bound on the order and computational complexity of the reconstruction. However, when the time-skew errors change, the coefficients of all 


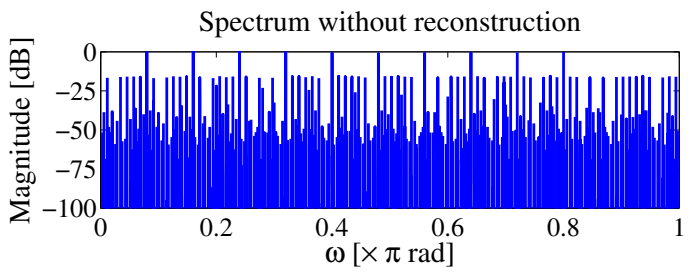

Spectrum after reconstruction

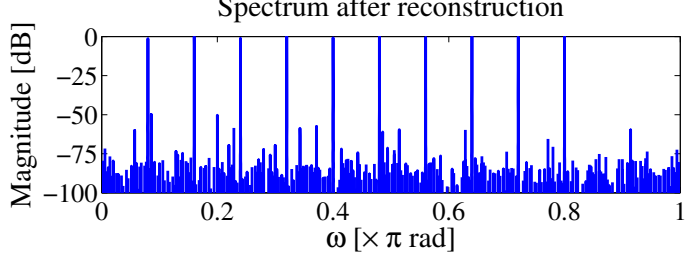

Fig. 3. Spectrum of the nonuniformly sampled TI-ADC output with missing samples, $y(n)$, and that of the reconstructed sequence, $\tilde{x}(n)$ using the constrained time-varying FIR reconstructor in the design example. The input to the TI-ADC is a multi-tone sinusoidal signal $x(n)=\sum_{r=1}^{10} \sin \left(n \omega_{r}\right)$, where $\omega_{r}=2 \pi r / 25$.

TABLE I

ORDER OF EACH FIR FILTER $h_{n}(k)$ FOR THE CONSTRAINED TIME-VARYING FIR RECONSTRUCTOR IN THE DESIGN EXAMPLE.

\begin{tabular}{|c|c|c|c|c|c|c|c|c|c|c|}
\hline $\boldsymbol{n}$ & $\mathbf{0}$ & $\mathbf{1}$ & $\mathbf{2}$ & $\mathbf{3}$ & $\mathbf{4}$ & $\mathbf{5}$ & $\mathbf{6}$ & $\mathbf{7}$ & $\mathbf{8}$ & $\mathbf{9}$ \\
\hline$N_{h_{n}}$ & 24 & 34 & 30 & 28 & 18 & 38 & 68 & 36 & 18 & 32 \\
\hline $\boldsymbol{n}$ & $\mathbf{1 0}$ & $\mathbf{1 1}$ & $\mathbf{1 2}$ & $\mathbf{1 3}$ & $\mathbf{1 4}$ & $\mathbf{1 5}$ & $\mathbf{1 6}$ & $\mathbf{1 7}$ & $\mathbf{1 8}$ & $\mathbf{1 9}$ \\
\hline$N_{h_{n}}$ & 30 & 28 & 24 & 68 & 36 & 28 & 16 & 32 & 30 & 34 \\
\hline $\boldsymbol{n}$ & $\mathbf{2 0}$ & $\mathbf{2 1}$ & $\mathbf{2 2}$ & $\mathbf{2 3}$ & $\mathbf{2 4}$ & $\mathbf{2 5}$ & $\mathbf{2 6}$ & $\mathbf{2 7}$ & & \\
\hline$N_{h_{n}}$ & 68 & 38 & 30 & 28 & 16 & 34 & 38 & 68 & & \\
\hline
\end{tabular}

the 28 FIR filters of this reconstructor need to be redetermined through 28 separate $R_{n} \times R_{n}$ matrix inversions. In the sub-band based reconstructor, when the time-skew error changes, only one $24 \times 24$ matrix should be inverted to compute the complex coefficients $c_{k m_{\ell}}$. Hence, compared to the time-varying reconstructor, the sub-band based reconstructor has lower online redesign complexity.

This is unlike the reconstructor in [1], where none of the filters have to be redesigned when the time-skew error changes. However, the order of the overall reconstructor is 396 with a computational complexity of around 550 multiplications per corrected output sample which is about 18 and 7 times higher than that of the constrained time-varying reconstructor and the sub-band based reconstructor, respectively. Also, it should be noted that the reconstructor in [1] uses a recursive structure which can limit the rate at which the reconstructor can operate. It can be seen from Table II that, for selecting the reconstruction scheme, there exists a trade-off between the design and the implementation complexity. The online design block is required only when a new set of $\varepsilon_{n}$ are estimated and

TABLE II

COMPARISON OF RECONSTRUCTOR COMPLEXITY FOR THE DESIGN EXAMPLE.

\begin{tabular}{|c|c|c|c|}
\hline Reconstructor & $\begin{array}{c}\text { Constrained } \\
\text { time-varying }\end{array}$ & $\begin{array}{l}\text { Sub-band } \\
\text { based }\end{array}$ & {$[1]$} \\
\hline Overall order & 68 & 960 & 396 \\
\hline $\begin{array}{l}\text { Average comp. } \\
\text { complexity }\end{array}$ & 31 & 75 & 550 \\
\hline $\begin{array}{l}\text { Online design } \\
\text { complexity }\end{array}$ & $\begin{array}{c}28 \text { matrix } \\
\text { inversions }\end{array}$ & $\begin{array}{l}1 \text { matrix } \\
\text { inversion }\end{array}$ & None \\
\hline Structure & Nonrecursive & Nonrecursive & Recursive \\
\hline
\end{tabular}
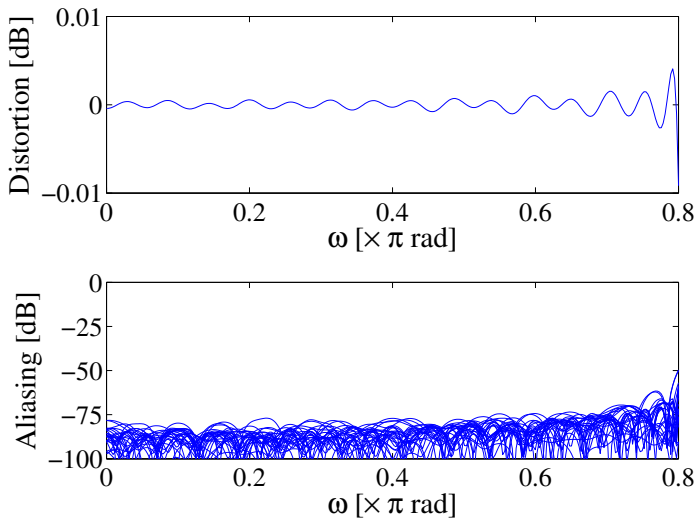

Fig. 4. Plot of the distortion function $V_{0}\left(e^{j \omega}\right)$ and the aliasing functions $V_{p}\left(e^{j \omega}\right), p=1,2, \ldots, 27$ for the constrained time-varying FIR reconstructor in the design example.

can be turned off after the new multiplier values are determined. In practice, the online design block is not used very often and hence, when compared to the reconstructor in [1], the reconstruction schemes presented here have significantly lower implementation complexity.

Figure 4 shows the distortion function $V_{0}\left(e^{j \omega}\right)$ and aliasing functions $V_{p}\left(e^{j \omega}\right), p=1,2, \ldots, 27$, for the constrained time-varying FIR reconstructor. The distortion and aliasing functions are evaluated using (54) and (55), respectively, in [6] which give the relation between $A_{n}(j \omega)$ and $V_{p}\left(e^{j \omega}\right), n, p=0,1, \ldots, 27$.

\section{CONCLUSION}

In this paper, we presented two nonrecursive reconstructors for TIADCs with missing samples. We also proposed least-squares based approach for the design of these two reconstructors. With the help of a design example, the design and the implementation complexities of the two reconstructors were compared. Also, we showed that, compared to existing recursive reconstructor, the reconstructors presented here achieve significantly lower computational complexity.

\section{REFERENCES}

[1] K. M. Tsui and S. C. Chan, "A novel iterative structure for online calibration of $M$-channel time-interleaved ADCs," IEEE Trans. Instrum. Meas., vol. 63, no. 2, pp. 312-325, Feb. 2014.

[2] M. Renfors and Y. Neuvo, "The maximum sampling rate of digital filters under hardware speed constraints," IEEE Trans. Circuits Syst., vol. 28 , no. 3, pp. 196-202, Mar. 1981.

[3] L. Zhao, X. Hu, C. Feng, S. Tang, S. Liu, and Q. An, "A 1.6-Gsps highresolution waveform digitizer based on a time-interleaved technique," IEEE Trans. Nucl. Sci., vol. 60, no. 3, pp. 2180-2187, 2013.

[4] T. Strohmer and J. Tanner, "Fast reconstruction algorithms for periodic nonuniform sampling with applications to time-interleaved ADCs," in Proc. IEEE Int. Conf. Acoustics, Speech Signal Process., vol. 3, 2007.

[5] S. Tertinek and C. Vogel, "Reconstruction of nonuniformly sampled bandlimited signals using a differentiator-multiplier cascade," IEEE Trans. Circuits Syst. I, vol. 55, no. 8, pp. 2273-2286, Sep. 2008.

[6] H. Johansson and P. Löwenborg, "Reconstruction of nonuniformly sampled bandlimited signals by means of time-varying discrete-time FIR filters," EURASIP J. Advances Signal Process., vol. 2006, Jan. 2006.

[7] A. K. M. Pillai and H. Johansson, "A sub-band based reconstructor for $M$-channel time-interleaved ADCs with missing samples," in Proc. IEEE Int. Conf. Acoustics, Speech Signal Process., May 2014.

[8] — - "Efficient recovery of sub-Nyquist CNUS sparse multi-band signals using analysis and modulated synthesis filter banks," in preparation.

[9] H. Johansson and A. Eghbali, "FIR filter with variable fractional delay and phase shift: Efficient realization and design using reweighted $\ell_{1}$-norm minimization," in Proc. IEEE Int. Symp. Circuits Syst., Beijing, China, May 19-23 2013. 